\title{
A small molecule that walks along a surface between porphyrin fences assembled in situ.**
}

\author{
Sam Haq, Bareld Wit, Hongqian Sang, Andrea Floris, Yu Wang, Jianbo Wang, Llü̈sa Pérez-García, \\ Lev Kantorovitch*, David B. Amabilino* and Rasmita Raval *
}

Dedication:

\begin{abstract}
A surface-constructed bimolecular system is described, comprising a simple divalent bis-imidazolyl molecule that is shown to "walk" in an inchworm fashion at room temperature along a specific pathway terminated at each end by oligomeric "fences" constructed on a monocrystalline copper surface. Scanning tunnelling microscopy shows the motion of the walker occurs along the $\left[\begin{array}{lll}1 & 1 & 0\end{array}\right]$ direction of the $\mathrm{Cu}$ surface with remarkably high selectivity, and is effectively confined by the orthogonal construction of covalent porphyrin oligomers along
\end{abstract}

[*] B. Wit, Dr. S. Haq and Prof. R. Raval

Surface Science Research Centre, Department of Chemistry University of Liverpool, Liverpool L69 3BX (United Kingdom) E-mail: raval@liv.ac.uk

Dr. L. Pérez-García

Laboratori de Química Orgànica, Facultat de Farmàcia, and Institut de Nanociència i Nanotecnologia

Universitat de Barcelona, 08028 Barcelona (Catalonia, Spain).

E-mail: mlperez@ub.edu

Prof. D.B. Amabilino

Institut de Ciència de Materials de Barcelona (CSIC)

Campus Universitari, 08193-Bellaterra (Catalonia, Spain).

Present address: School of Chemistry, The University of

Nottingham, University Park, Nottingham NG7 2RD (United

Kingdom) E-mail: David.Amabilino@nottingham.ac.uk

Prof. L. Kantorovich, Dr. A. Floris

Physics Department, King's College London, Strand,

London, SE2 OLR, United Kingdom.

E-mail lev.kantorovitch@kcl.ac.uk

H. Sang, Prof. Y. Wang and Prof. J. Wang

School of Physics and Technology, Center for Electron Microscopy and MOE Key Laboratory of Artificial Micro- and Nano-structures, Wuhan University, Wuhan 430072, China

E-mail: yu.wang@whu.edu.cn (YW); wang@whu.edu.cn (JW)

[**] This research was supported by the UK EPSRC grants EP/ J019844/1 and EP/F00981X/1, the MICINN (TEC2011-29140-C0302). HS, YW and JW acknowledge the financial support from the 973 Program (2011CB933300), the National Natural Science Foundation of China (11328403, 51271134 and J1210061), the Fundamental Research Funds for the Central Universities, and the CERS-1-26 (CERS-China Equipment and Education Resources System).

Supporting information for this article is available on the WWW under http://dx.doi.org/10.1002/anie.201xxxxxx the [001] surface direction, which serve as barriers. Density functional theory shows that the mobile molecule walks by attaching and detaching the nitrogen atoms in its imidazolyl "legs" to and from the protruding close-packed rows of the metal surface and transiting via a small energy barrier between two energetically equivalent extended and contracted conformations.

$\boldsymbol{W}_{\text {alking molecules }}{ }^{[1]}$ have the potential to perform a variety of functions, from molecular transport, motility, to energy conversion. ${ }^{[2]}$ A key goal for artificial systems is confining molecular motion between two or more locations along a specified path. Biology, for example, achieves this with linear protein motor systems, such as myosin on actin or kinesin on microtubule filaments. ${ }^{[3]}$ Surfaces are especially relevant environments to build and study molecular machines. ${ }^{[4]}$ In particular, the formation of dynamic molecular systems that display motion along a given path on surfaces is an area of intense interest. ${ }^{[5]}$ The non-directional ${ }^{[6]}$ and uni-directional ${ }^{[7]}$ motion of "molecular cars" on different surfaces are impressive achievements, with motion induced by stimuli. For surface-based molecular machines, important next steps include the creation of simple walking molecules, operation at room temperature and the confinement of motion within suitable surface frameworks. ${ }^{[8]}$

Here we show a bi-component surface-based system wherein a simple molecular "walker" - facilitated by suitable conformations and specific interaction with the surface - leads to directional walking motion via attachment and detachment at certain locations on a finite track akin to an inchworm mechanism. ${ }^{[3 b]}$ Furthermore, this directional motion can be effectively confined by construction of covalent molecular fences on the metal, which are specifically grown along a perpendicular direction to the path. In essence, the preferred path of the walker is now confined between two barriers, in analogy to an abacus. ${ }^{[9]}$ In the type of system we describe at least two molecule-based components are necessary: One is immobile, and acts as a confining barrier (the "fence") to the motion of the second component (the "walker"). The latter component must move quickly and predominantly along one path dictated by specific interactions with an anisotropic single crystal surface as illustrated schematically in Figure 1(i).

The immobile molecular component - the "fence" - was formed from cobalt(II) porphyrin 1 (Figure 1(ii)), which generates covalent oligomeric organometallic chains when heated on the $\mathrm{Cu}(110)$ surface, as demonstrated for analogous compounds. ${ }^{[10]}$ Scanning tunnelling microscopy (STM) shows that the distance between the neighbouring monomeric units within the chain is $1.08 \mathrm{~nm}$, which is 
(i)

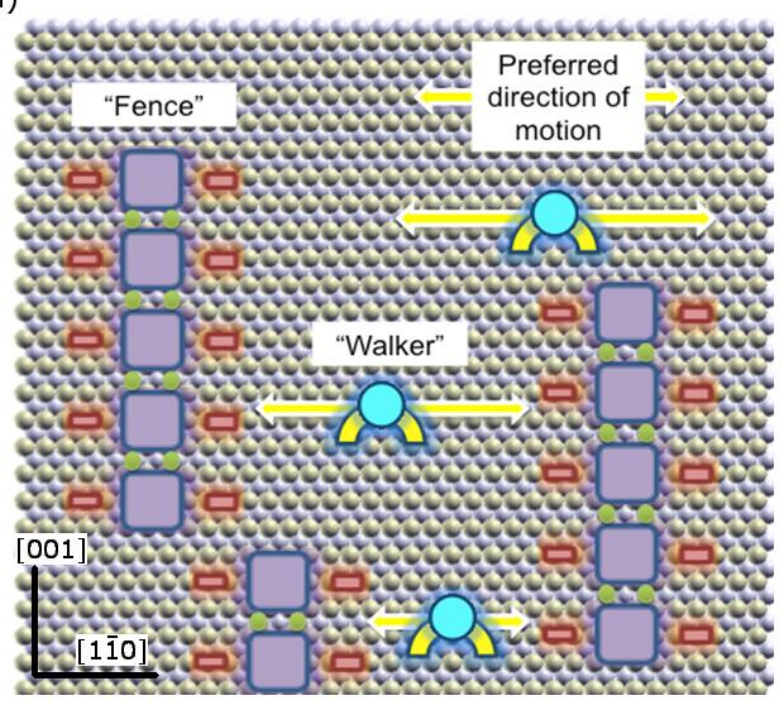

(ii)

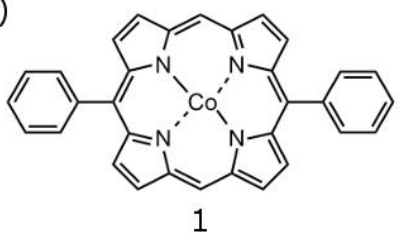

(iii)
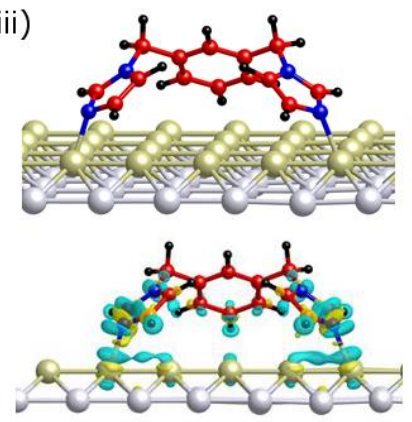

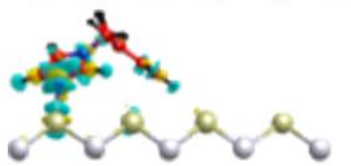

Extended

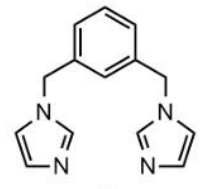

2
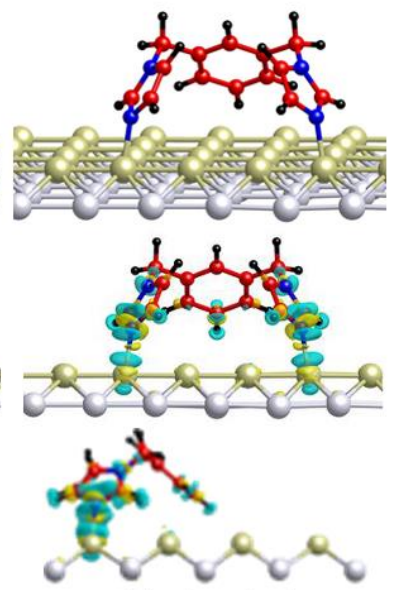

Contracted

(iv)

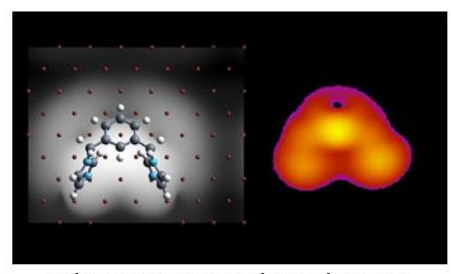

Theory: Simulated STM

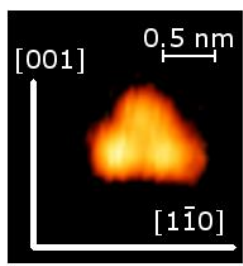

Experiment
Figure 1. (i) Representation of the system described in this paper, in which a mobile molecule (a "walker") shuttles between two immobile barriers ("fences") along a preferred direction of diffusion due to the anisotropy of the underlying single crystal surface. (ii) Cobalt-diphenylporphyrin (molecule 1) and 1,3bis(imidazol-1-yl methyl)benzene (molecule 2), which form the immobile and mobile components of the system, respectively. (iii) Two DFT relaxed energetically equivalent geometries of the molecule on the surface with the feet held four (Extended) and three (Contracted) $\mathrm{Cu}$ atomic distances $\left(\mathrm{a}_{0}\right)$ apart shown as: (upper panel) ball-and-stick models of the calculated geometries: (middle panel) front and (bottom panel) side views of the structures with the charge density difference shown in yellow (excess) and green (depletion) colours at \pm 0.003 a.u. (iv) Simulated STM $(1.0 \mathrm{~V})$ of the contracted geometry and experimental STM image $(I(t)=-0.14 n A, V(t)=-710 m V)$ of a single molecule on the surface. consistent with edge-to-edge porphyrin-Cu-porphyrin connections, created via dehydrogenation of one, two or three $s p^{2} \mathrm{C}-\mathrm{H}$ bond(s) at the 3,5 and 7 positions and subsequent formation of $\mathrm{C}-\mathrm{Cu}-\mathrm{C}$ bonds. ${ }^{[10]}$ The fences run specifically along the highly corrugated [001] direction of the $\mathrm{Cu}$ surface $^{[10]}$ and are immobile at room temperature, thus acting as an effective barrier to any molecular motion perpendicular to them. The perpendicular [11 0] direction of the surface has close-packed rows of copper atoms running along it, which turns out to be an ideal path for the walker molecules to move along, as described below.

Compound 2 (Figure 1(ii)) was used as the walker molecule, because it provided two particular attributes: first, the ability to bind to the surface with reasonable strength via specific molecule- $\mathrm{Cu}$ interactions; and, second, possessing sufficient conformational flexibility to allow binding to surface atoms located at different separations, thus enabling the molecule to shift position without large energetic penalties. The walker molecule $\mathbf{2}$ has two "feet" that adhere specifically to the surface; here, the imidazolyl groups perform this function because the $\mathrm{N}$ atoms can adsorb specifically and reversibly to the $\mathrm{Cu}$ atoms in the top row of the surface. A degree of flexibility is built into the molecule via the methylene spacer in between the imidazolyl groups and the phenyl ring. In solution, this spacer allows the formation of a horseshoe-shaped conformation when appropriate supramolecular interactions take place $^{[11]}$, while in the solid state the derivative's legs are usually splayed. ${ }^{[12]}$ These observations are clear indicators of the flexibility of the molecule that would make it amenable for a walking-type motion. Specifically, the horseshoe conformation is ideal for binding to the surface via both imidazolyl feet, while the skeletal flexibility enables the molecule to occupy different adsorption geometries, allowing its feet to bind different surface atoms.

Periodic density functional theory (DFT) calculations of the walker molecule on the $\mathrm{Cu}(110)$ surface confirm that the molecule adopts the horseshoe conformation, in which the phenyl ring is held up away from the surface and the molecule stands on its two imidazolyl feet that are held to the surface via chemisorption bonds between the two pyridyl-like nitrogen atoms and the surface $\mathrm{Cu}$ atoms, as shown in the charge density difference plot Figure 1(iii). In this stance, the molecule may adopt several stable geometries with binding energies ranging between 325 and $348 \mathrm{~kJ} / \mathrm{mol}$ (see Supporting Information: Figure S1). The two most favourable structures, with identical adsorption energies of $348 \mathrm{~kJ} / \mathrm{mol}$, are found when the two imidazolyl feet are adsorbed on the same closepacked $\mathrm{Cu}$ row, separated by either four or three $\mathrm{Cu}$ atomic distances $\left(\mathrm{a}_{0}\right)$, Figure 1(iii), labelled as the Extended and Contracted conformations, respectively. STM images of the walker molecule obtained when diffusion is arrested at either low temperatures or when trapped between fences consistently show a three-lobed structure, with two lobes aligned along the close-packed [11 0 ] direction (Figure 1(iv)). This experimental STM image is closely matched by the simulated STM image of the calculated walker adsorbed with its feet held three $\mathrm{Cu}$ atoms apart, Figure 1(iv), with the three imaged lobes corresponding to the three rings present in the molecular structure of $\mathbf{2}$.

When the walker molecule $\mathbf{2}$ was deposited on the clean $\mathrm{Cu}(110)$ surface, long, straight, bright lines traversing tens of nanometres were observed at $135 \mathrm{~K}$, suggesting unhindered and strongly anisotropic directional motion along the close-packed [11 0] direction, Figure 2(i). The bright diffusion tracks do not seem to change direction frequently in a single image, and have dimensions far larger than those of molecule $\mathbf{2}$, which is imaged as being approximately $1 \mathrm{~nm}$ long and $0.8 \mathrm{~nm}$ wide, Figure 1(iv). Therefore the bright lines correspond to directional motion of the molecules, which is fast on the timescale of the STM raster scanning. 


\section{Angewandte Communications}

(i)

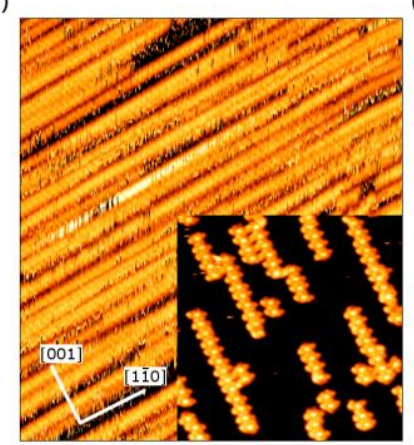

(iii)

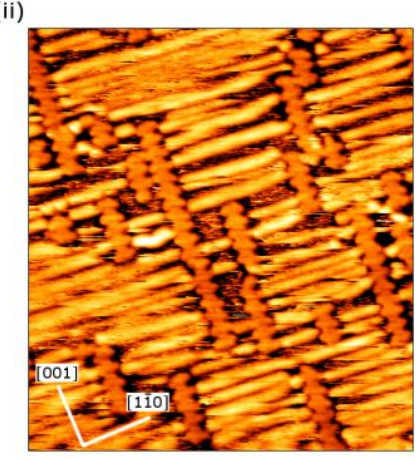

State 3

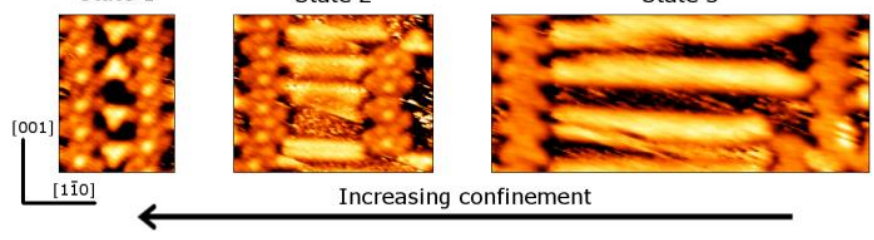

Figure 2. (i) A representative STM image at $135 \mathrm{~K}$ of molecule 2 diffusing on the $\mathrm{Cu}(110)$ surface $\left(34.4 \times 41.7 \mathrm{~nm}^{2}, \mathrm{I}(\mathrm{t})=-0.21 \mathrm{nA}, \mathrm{V}(\mathrm{t})=-390 \mathrm{mV}\right)$ and (inset) STM image at RT of fences of $1\left(21.6 \times 23.7 \mathrm{~nm}^{2}, \mathrm{I}(\mathrm{t})=-0.12 \mathrm{nA}, \mathrm{V}(\mathrm{t})=\right.$

$1360 \mathrm{mV}$ ). (ii) A representative STM image at RT of 2 diffusing between fences of $\mathbf{1}$, created by first forming fences on $\mathrm{Cu}(110)$ with 2 deposited subsequently $\left(33.1 \times 40.0 \mathrm{~nm}^{2}, \mathrm{I}(\mathrm{t})=0.32 \mathrm{nA}, \mathrm{V}(\mathrm{t})=780 \mathrm{mV}\right)$. Coordinates apply to inset of (i) as well. (iii) Three close up STM images at RT of molecules 2 in between fences of 1 that are placed at different distances with respect to each other, leading to different degrees of confinement (State $1: 5.8 \times 7.1 \mathrm{~nm}^{2}, \mathrm{I}(\mathrm{t})=-0.18$ $\mathrm{nA}, \mathrm{V}(\mathrm{t})=-1250 \mathrm{mV}$, State 2: $8.2 \times 7.1 \mathrm{~nm}^{2}, \mathrm{I}(\mathrm{t})=-0.18 \mathrm{nA}, \mathrm{V}(\mathrm{t})=-1200 \mathrm{mV}$ State 3: $\left.15.5 \times 7.1 \mathrm{~nm}^{2}, \mathrm{I}(\mathrm{t})=0.32 \mathrm{nA}, \mathrm{V}(\mathrm{t})=780 \mathrm{mV}\right)$. Coordinates apply to all three states.

In order to create confined motion, oligomeric fences of $\mathbf{1}$ were pre-formed on the surface to create barriers that lie orthogonal to the walker's diffusion direction, Figure 1(i) and 2(i, inset). After deposition of molecule 2 onto the fence-strewn metal surface, STM images obtained at room temperature show the original immobile lines of linked porphyrin fences and, between them, bright lines corresponding to high tunnelling current (Figure 2(ii)). These bright lines run perpendicularly to the porphyrin 1 fences, demonstrating that the movement of the molecules of $\mathbf{2}$ is still predominantly in the

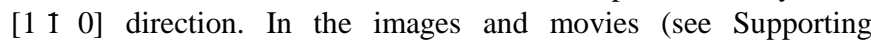
information: Figures S2-S3) there are never indications that the mobile adsorbate can jump onto or over the fences, as we do not see any static walker molecules or noise in the STM images, corresponding to molecular motion, on top of the porphyrin fences, which are always imaged clearly. We, therefore, conclude that we are witnessing constrained surface Brownian motion essentially in one dimension dictated by the interaction of the molecule $\mathbf{2}$ with the surface and confined by the porphyrin fences.

It is clear that the mobility of the molecules of $\mathbf{2}$ is restricted by the porphyrin fences, which are separated from one another at random distances and at random positions on the surface. Thus, depending upon where the fences happen to be located, the walker molecules have free straight paths of varying length to travel along, as seen in the large area image shown in Figure 2(ii). More detail on the diffusion behaviour as the confinement is increased is shown in Figure 2(iii).

When the porphyrin fences are so close that perpendicular diffusion of a potentially dynamic molecule is not possible, then the latter are trapped so that they can be imaged individually by STM as three lobed structures, State 1 in Figure 2(iii). If the chains are positioned a little further apart, so that the interspersed surface space increases by a few atomic distances, then the walker molecules diffuse between the porphyrin chains, State 2 in Figure 2(iii). In such restricted spaces, the diffusion lines often show different intensity patterns corresponding to different residence time of $\mathbf{2}$ in a given region. For example, the middle two diffusion tracks in State 2, Figure 2(iii), show enhanced intensity at the two ends close to the fences, suggesting a greater residence time of the walker molecule near the fences. As the distance between the fences is increased, the diffusion of the walker lengthens accordingly, State 3 in Figure 2(iii).

The walkers are also occasionally imaged next to the long edge of the fence without being trapped, suggesting a locally favourable adsorption site of $\mathbf{2}$ so as to reduce its diffusion (see Supplementary Information: Figure S3). Additionally, there appears to be favourable interactions with the short edge of the fence. Thus, these short edges can temporarily trap the walker molecules if they diffuse past in close proximity (Figure 3(i)). This effect is especially strong if two fences are positioned close together in the [001] direction, providing small opening spaces, which the walker can diffuse through. For example, the walker molecules can pass through gaps as small as $1.5 \mathrm{~nm}$ in the fence, but only at a significantly reduced rate (Figure 3(ii)). In figurative terms, the space between the fence acts like a turnstile for the walker molecules.

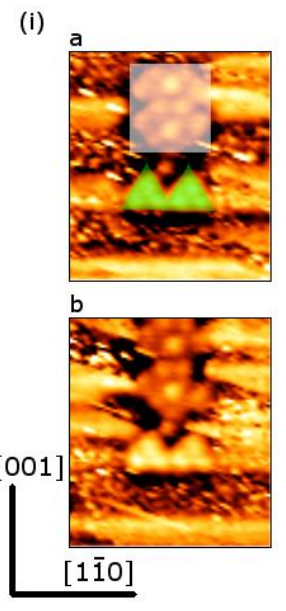

(ii)
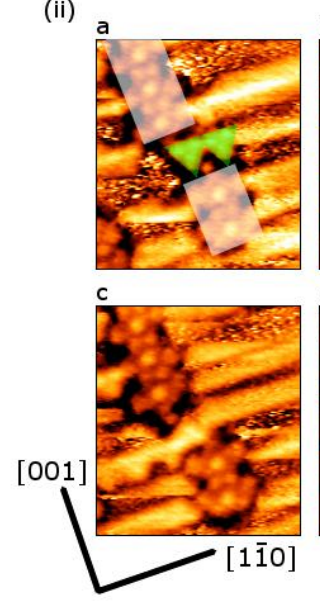
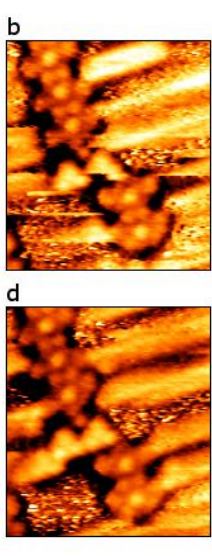

Figure 3. (i) Two $4.8 \times 5.5 \mathrm{~nm}^{2}$ STM images at RT of two molecules 2 trapped temporarily at the short edge of a fence of $1(\mathrm{a}: \mathrm{I}(\mathrm{t})=-0.15 \mathrm{nA}, \mathrm{V}(\mathrm{t})=-1200 \mathrm{mV}$ b: $\mathrm{I}(\mathrm{t})=-0.19 \mathrm{nA}, \mathrm{V}(\mathrm{t})=-1200 \mathrm{mV}$ ). (ii) Four subsequent $6.9 \times 8.5 \mathrm{~nm}^{2} \mathrm{STM}$ images of multiple molecules of 1 moving through a small gap of approximately $1.5 \mathrm{~nm}$ in between two chains of $2(\mathrm{a}: \mathrm{I}(\mathrm{t})=-0.16 \mathrm{nA}, \mathrm{V}(\mathrm{t})=-1200 \mathrm{mV}, \mathrm{b}: \mathrm{l}(\mathrm{t})=$ $0.17 \mathrm{nA}, \mathrm{V}(\mathrm{t})=-1200 \mathrm{mV}, \mathrm{c}: \mathrm{I}(\mathrm{t})=-0.20 \mathrm{nA}, \mathrm{V}(\mathrm{t})=-1200 \mathrm{mV}, \mathrm{d}: \mathrm{I}(\mathrm{t})=-0.18 \mathrm{nA}$, $\mathrm{V}(\mathrm{t})=-1200 \mathrm{mV})$. The locations of the chains and two walker molecules are highlighted in the first images of (i) and (ii).

In light of these results and in order to understand how the adsorbed walker molecule can diffuse on the surface, nudged elastic band calculations were carried out to bridge various adsorption geometries along a given travel path. Diffusion of several starting conformations was studied in detail (see Supporting information: Figures S4-S8) including the most favourable one in which the molecule stands with two feet on the same row (Figures 1(iii)). Dynamic modelling reveals that the lowest diffusion barriers occur when the molecule adopts its most favoured conformations with both feet on the same row and walks along the surface rows with an inchworm motion (Figure 4(i)), involving a low energy barrier of only $16 \mathrm{~kJ} / \mathrm{mol}$. There are specific aspects involved in this walk as shown in Figure 4(i): first, starting from the energetically favoured Extended conformation, the molecule moves its rear "leg" to the nearest $\mathrm{Cu}$ atom along the row, thereby forming a higher energy transient structure with legs three $\mathrm{Cu}$ atomic distances apart, before relaxing into the almost equally favoured Contracted conformation. Then the forward "leg" steps forward bonding to the nearest $\mathrm{Cu}$ atom, again yielding a higher energy transient structure, before forming the final extended configuration equivalent to the initial one but now advanced forward by one surface atom. The transition from 
the Extended to Contracted conformations (or vice versa) requires breaking one $\mathrm{N}-\mathrm{Cu}$ bond and moving the leg to the adjacent $\mathrm{Cu}$ atom, where a new $\mathrm{N}-\mathrm{Cu}$ bond is made, and the walker assumes a transitional conformation that is some $12-16 \mathrm{~kJ} / \mathrm{mol}$ higher, before relaxing to the appropriate favoured conformation. The evolution of electron density during a bond-breaking process is shown in Figure 4(i). The conformational flexibility of the walker molecule and the match of the molecular dimensions to the underlying surface ensure that such a walking process is not energetically expensive if carried out along the close-packed [11 0] direction. This is consistent with the fact that, during the diffusion process, the $\mathrm{Cu}$ atoms displace by no more than $0.001 \mathrm{~nm}$. We note, that, the probability of the front leg or the rear leg moving first in opposite directions are identical,

\section{(i) Motion along the $[1 \overline{1} 0]$ direction by an inchworm walking mechanism}
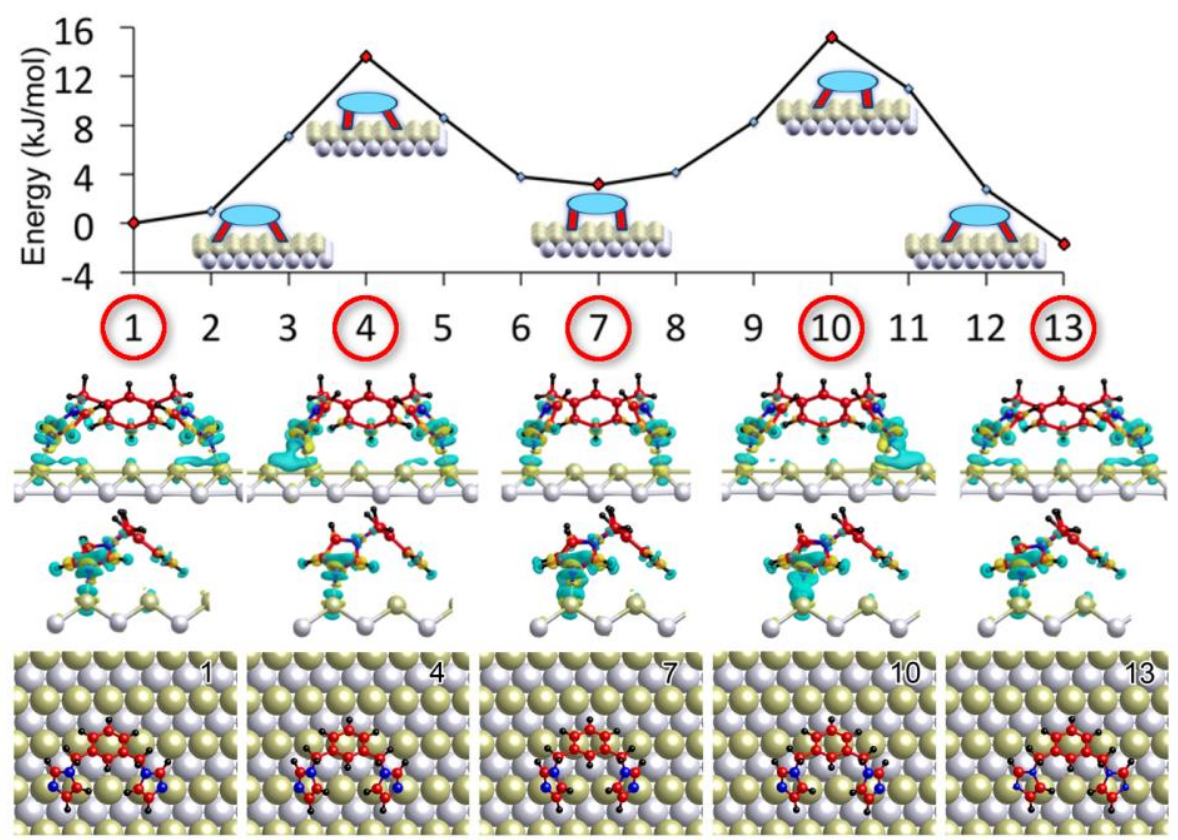

(ii) Motion along the [001] direction by a pivoting walking mechanism

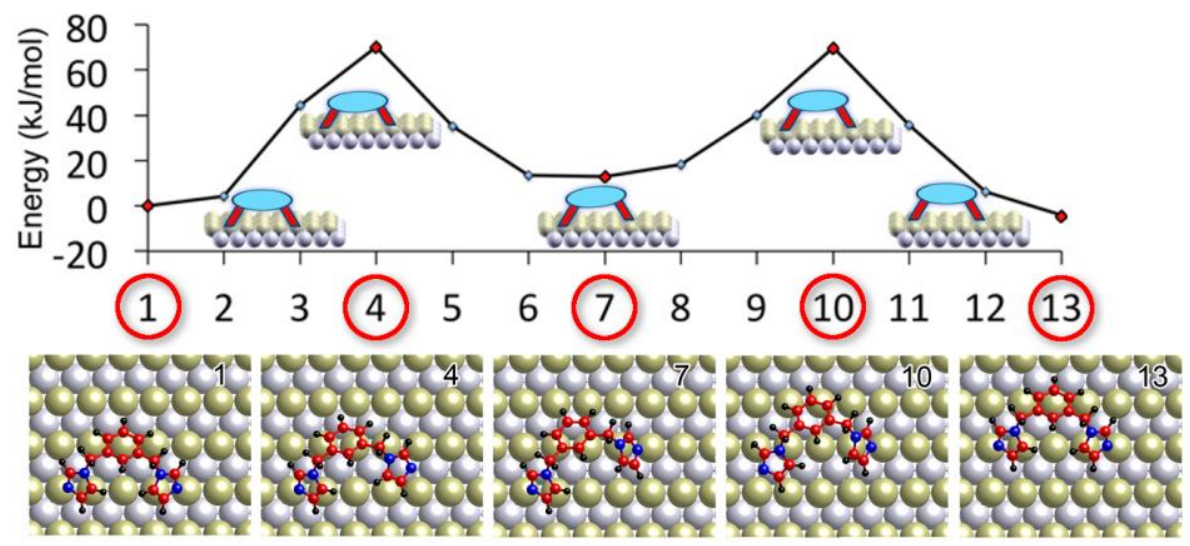

Figure 4. (i) The DFT calculated minimum energy path, and the corresponding front, side and top views of the selected geometries, for molecule 2 to diffuse one lattice constant $a_{0}$ along the $\mathrm{Cu}$ row between two equivalent Extended structures. The molecule first steps with its rear leg towards its front one to the nearest $\mathrm{Cu}$ atom (points 1 to 7 ) to arrive into the Contracted geometry, which is followed by the front leg to make another step by $a_{0}$ in the same direction to arrive into equivalent (but shifted by $a_{0}$ ) Extended geometry (points 7 to 13). (ii) The DFT calculated minimum energy path, and the corresponding top views of the selected geometries, for molecule 2 to diffuse one lattice constant $b_{0}$ perpendicular to the Cu row between two equivalent Extended structures. The molecule first pivots on its rear leg to get the front leg onto the next row (points 1 to 7), then it pivots on the front leg to get the rear leg across (points 7 to 13). The charge density differences plots are shown for all selected structures in (i) using the same colour scheme as in Figure 1. complex diffusion path with the largest barrier of nearly $50 \mathrm{~kJ} / \mathrm{mol}$ separates the two geometries in which a molecule oriented along the close-packed $\mathrm{Cu}$ rows transforms to an orthogonal orientation across the $\mathrm{Cu}$ rows (Figure S8); we expect this would be a rare event at room temperature.

Overall, our dynamic modelling agrees with the experimental observations that the preferred diffusion direction is along the rows, with the diffusion rate in the orthogonal direction across the rows calculated to be at least $10^{5}$ times smaller at room temperature. This selectivity in direction of diffusion is due to the specific manner in which the molecule binds to the surface and the anisotropic structure of the surface, leading to different barriers for motion in the two orthogonal directions.

In conclusion, we show that a simple molecule can walk on a preferred path on a surface, and that this motion can be effectively restricted between two positions via on-surface synthesized porphyrin oligomer fences aligned perpendicular to the walking direction. Thus, the simple imidazolyl walker molecule shuttles back and forth between the fences along the close-packed metallic rows, which act as intrinsic and directional tracks on the corrugated $\mathrm{Cu}(110)$ substrate. DFT modelling gives unique insight into this process and shows that the directionality of motion arises from the specific interaction of the walker with the surface, which moves by attaching and detaching its imidozolyl feet in an inchworm fashion, and from anisotropic diffusion barriers that favour this motion along a preferred direction. Thus, an anisotropic surface such as $\mathrm{Cu}(110)$ is an advantageous medium to place molecular machines, used by others in other contexts, ${ }^{[13-18]}$ with inherent structural features providing tracks along which molecules could be confined to shuttle. We note that the porphyrin framework can be readily functionalized at the phenyl positions of $\mathbf{1}$, which brings further 
prospects for creating non-equivalent 'stations' for the walker to traverse between, and also for engineering specific recognition of the shuttling molecule at either or both stations. Our system operates under thermal activation (room temperature is sufficient here), but it is also conceivable that systems could be designed where shuttling is initiated by, say, an electrical pulse or light activation, where aspects such as microscopic reversibility ${ }^{[19]}$ could be studied in detail at the submolecular level. Finally, dissymmetry in the walker mobility could lead to uni-directional motion; we are presently working towards these targets.

\section{Experimental Details.}

The experiments were carried out in a UHV STM setup $\left(P<2 \cdot 10^{-10} \mathrm{mbar}\right)$. The $\mathrm{Cu}(110)$ crystal was cleaned using repeated argon sputtering and annealing cycles. Compounds 1 and $\mathbf{2}$ were sublimed onto the $\mathrm{Cu}(110)$ crystal at RT. STM data was obtained using a Specs Aarhus 150 STM operated in constant current mode. Images were analysed using the Gwyddion 2.30 software package. $^{[20]}$

DFT calculations were performed using the CP2K Quickstep package ${ }^{[21]}$ which implements a hybrid Gaussian (optimised double-zeta plus polarization, DZVP-MOLOPT) and plane wave (300 Ry cutoff) basis set, Goedecker-TeterHutter pseudopotentials ${ }^{[22]}$ and the Perde-Burke-Ernzerhof (PBE) exchangecorrelation density functional. ${ }^{[23]}$ The van der Waals interaction was approximately taken into account within the Grimme D3 method. ${ }^{[24]}$ The force convergence criterion in geometry relaxation was $0.01 \mathrm{eV} / \AA$. The $\mathrm{Cu}(110)$ surface was modelled using 4 layers of $\mathrm{Cu}$ atoms with the two bottom layers fixed in the bulk-like positions, and a vacuum gap of $15 \AA$. The counterpoise correction was used to remove the basis set superposition error (BSSE) from the calculated adsorption energies ${ }^{[25]}$ Climbing image nudged elastic band $(\mathrm{Cl}$ NEB) calculations ${ }^{[26]}$ were performed typically with 7 replicas connecting initial and final band points. STM images were obtained by calculating the integrated local density of states within the Tersoff-Hamann approximation. ${ }^{[27]}$ Some calculations were also conducted using VASP ${ }^{[28]}$ and Quantum ESPRESSO plane wave codes ${ }^{[29]}$ to check some of the adsorption geometries and energies.

Received: ((will be filled in by the editorial staff))

Published online on ((will be filled in by the editorial staff))

Keywords: Bottom-up • Dynamic • Nanomaterials • Diffusion • Molecular machine

[1] M. von Delius, D.A. Leigh, Chem. Soc. Rev., 2011, 40, 3656-3676.

[2] A. Coskun, M. Banaszak, R.D. Astumian, J.F. Stoddart, B.A. Grzybowski, Chem. Soc. Rev., 2012, 41, 19-30.

[3] (a) D.J.G. Bakewell, D.V. Nicolau, Aust. J. Chem., 2007, 60, 314-332. (b) S. S. Patel, I. Donmez, J. Biol. Chem., 2006, 281, 18265-18268.

[4] (a) D. Lensen, J.A.A.W. Elemans, Soft Matter., 2012, 8, 9053-9063. (b) S. Ishihara, Y. Wakayama, N. Hiroshiba, J.P. Hill, K. Ariga, Curr. Org. Synth., 2012, 9, 428-438. (c) J. Puigmartí-Luis, W.J. Saletra, A. González, D.B. Amabilino, L. Pérez-García, Chem. Commun., 2014 50, 82-84. (d) J. Bern, D. A. Leigh, M. Lubomska, S. M. Mendoza, E. M. Perez, P. Rudolf, G. Teobaldi, F. Zerbetto, Nat. Matter., 2005, 4, 704-710.

[5] (a) H. Hess, C.M. Matzke, R.K. Doot, J. Clemmens, G.D. Bachand, B.C. Bunker, V. Vogel, Nano Lett., 2003, 3, 1651-1655. (b) K.Y. Kwon, K.L. Wong, G. Pawin, L. Bartels, S. Stolbov, T.S. Rahman,
Phys. Rev. Lett., 2005, 95, 166101. (c) Y. Makoudi, E. Arras, N. Kepčija, W. Krenner, S. Klyatskaya, F. Klappenberger, M. Ruben, A.P. Seitsonen, J.V. Barth, ACS Nano, 2012, 6, 549-556.

[6] Y. Shirai, A.J. Osgood, Y. Zhao, K.F. Kelly, J.M. Tour, Nano Lett., 2005, 5, 2330-2334

[7] T. Kudernac, N. Ruangsupapichat, M. Parschau, B. Macia, N. Katsonis, S.R. Harutyunyan, K.H. Ernst, B.L. Feringa, Nature, 2011, 479, 208-211.

[8] D. Kühne, F. Klappenberger, W. Krenner, S. Klyatskaya, M. Ruben, J.V. Barth, Proc. Natl. Acad. Sci. USA, 2010, 107, 21332-21336.

[9] (a) M. T. Cuberes, R. R. Schlittler, J. K. Gimzewski, Appl. Phys. Lett. 1996, 69 , 3016-3018. (b) H. Shigekawa, K. Miyake, J. Sumaoka, A. Harada, M. Komiyama, J. Am. Chem. Soc., 2000, 122, 5411-5412

[10] (a) S. Haq, F. Hanke, M.S Dyer, M. Persson, P. Iavicoli, D.B. Amabilino, R. Raval, J. Am. Chem. Soc., 2011, 133, 12031-12039. (b) F. Hanke, S. Haq, R. Raval, M. Persson, ACS Nano, 2011, 5, 90939103. (c) S. Haq, F. Hanke, J. Sharp, M. Persson, D.B. Amabilino, R. Raval, ACS Nano, 2014, 8, 8856-8870.

[11] S. Ramos, E. Alcalde, G. Doddi, P.Mencarelli,L. Perez-Garcia, J. Org. Chem., 2002, 67, 8463-8468

[12] E. Alcalde, N. Mesquida, M. Alemany, C. Alvarez-Rua, S. GarciaGranda, P. Pacheco, L. Perez-Garcia, Eur. J. Org. Chem., 2002, 12211231.

[13] M. Schunack, T.R. Linderoth, F. Rosei, E. Laegsgaard, I. Stensgaard, F. Besenbacher, Phys. Rev. Lett., 2002, 88, 156102

[14] R. Otero, Y. Naitoh, F. Rosei, P. Jiang, P. Thostrup, A. Gourdon, E. Laegsgaard, I. Stensgaard, C. Joachim, F. Besenbacher, Angew. Chem. Int. Ed., 2004, 43, 2092-2095

[15] R. Otero, F. Hummelink, F. Sato, S.B. Legoas, P. Thostrup, E. Laegsgaard, I. Stensgaard, D.S. Galvao, F. Besenbacher, Nature Mater., 2004, 3, 779-782.

[16] K.L. Wong, G. Pawin, K.Y. Kwon, X. Lin, T. Jiao, U. Solanki, R.H.J. Fawcett, L. Bartels, S. Stolbov, T.S. Rahman, Science, 2007, 315, 1391-1393.

[17] S.C. Jensen, A. Shank, R.J. Madix, C.M. Friend, ACS Nano, 2012, 6 2925-2930.

[18] X. Bouju, F. Cherioux, S. Coget, G. Rapenne, F. Palmino, Nanoscale, 2013, 5, 7005-7010.

[19] R.D. Astumian, Nat. Nanotechnol., 2012, 7, 684-688.

[20] D. Nečas, P. Klapetek, Cent. Eur. J. Phys., 2012, 10, 181-188.

[21] J. Hutter, M. Iannuzzi, F. Schiffmann, J. Van deVondele, WIREs Comput. Mol. Sci., 2013, 4, 15-25.

[22] S. Goedecker, M. Teter, J. Hutter, Phys. Rev., B Condens. Matter., 1996, 54, 1703-1710

[23] J. P. Perdew, K. Burke, M. Ernzerhof, Phys. Rev. Lett., 1996, 77, 3865-3868

[24] S. Grimme, J. Antony, S. Ehrlich, H. Krieg, J. Chem. Phys., 2010, 132, 154104.

[25] S. Boys, F. Bernardi, Mol. Phys., 1970, 19, 553-566.

[26] G. Henkelman, B. P. Uberuaga, H. Jónsson, J. Chem. Phys., 2000, 113, 9901-9904.

[27] J. Tersoff, D. R. Hamann, Phys. Rev. B, 1985, 31, 805-813

[28] G. Kresse, J. Furthmüller, Phys. Rev. B, 1996, 54, 11169-11186.

[29] P. Giannozzi, S. Baroni, N. Bonini, M. Calandra, R. Car, C. Cavazzoni, D. Ceresoli, G.L Chiarotti, M. Cococcioni, I. Dabo, A. Dal Corso, S. de Gironcoli, S. Fabris, G. Fratesi, R. Gebauer, U. Gerstmann, C. Gougoussis, A. Kokalj, M. Lazzeri, L. Martin-Samos, N. Marzari, F. Mauri, R. Mazzarello, S. Paolini, A. Pasquarello, L. Paulatto, C. Sbraccia, S. Scandolo, G. Sclauzero, A.P Seitsonen, A. Smogunov, P. Umari, R.M. Wentzcovitch, J. Phys.: Condens. Matter, 2009, 21, 395502. 


\section{Angewandte \\ Communications}

Entry for the Table of Contents (Please choose one layout)

Layout 1:

\section{Walking the line}

Sam Haq, Bareld Wit, Hongqian Sang, Andrea Floris, Yu Wang, Jianbo Wang, Lluïsa Pérez-García, Lev Kantorovitch,* David B. Amabilino *and Rasmita Raval* Page - Page

A small molecule that walks along a surface between porphyrin fences assembled in situ.

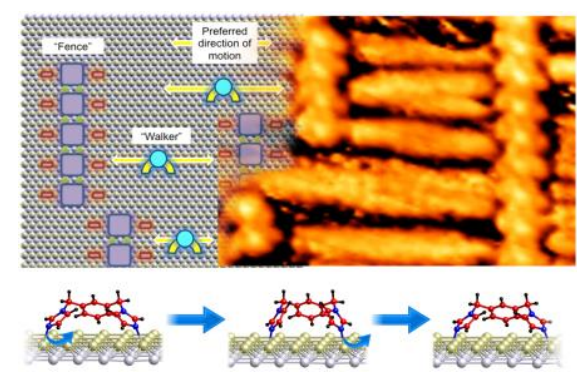

A bimolecular system was constructed from a bis-imidazolyl compound and porphyrin derivative on an anisotropic monocrystalline copper surface. The former is mobile and is shown to "walk" along one preferred surface direction with remarkably high selectivity. The latter can be oligomerized to form "fences" orthogonal to the walking direction of the former. These fences act as an insurmountable barriers for the walker and effectively confine its motion. 